\title{
Opinion Paper
}

Giuseppe Lippi ${ }^{\mathrm{a}, \star}$, Mauro Panteghini ${ }^{\mathrm{a}}$, Sergio Bernardini ${ }^{\mathrm{a}}$, Laura Bonfanti ${ }^{\mathrm{b}}$, Paolo Carraro ${ }^{\mathrm{a}}$, Ivo Casagranda ${ }^{\mathrm{b}}$, Mario Cavazza ${ }^{\mathrm{b}}$, Ferruccio Ceriotti ${ }^{\mathrm{a}}$, Marcello Ciaccio ${ }^{\mathrm{a}}$, Daniele Coen ${ }^{\mathrm{b}}$, Davide Giavarina ${ }^{a}$, Fabrizio Giostra ${ }^{b}$, Ciro Paolillo ${ }^{b}$, Mario Plebania ${ }^{a}$, Giorgio Ricci ${ }^{b}$ and Gianfranco Cervellin ${ }^{b}$

\section{Laboratory testing in the emergency department: an Italian Society of Clinical Biochemistry and Clinical Molecular Biology (SIBioC) and Academy of Emergency Medicine and Care (ACEMC) consensus report}

DOI 10.1515/cclm-2017-0077

Received January 27, 2017; accepted March 3, 2017

\begin{abstract}
Background: The mainstay of patient-oriented laboratory testing in emergency settings entails selecting a number and the type of tests according to valid criteria of appropriateness. Since the pattern of urgent tests requesting is variable across different institutions, we designed a joined survey between the Academy of Emergency Medicine and Care (AcEMC) and the Italian Society of Clinical Biochemistry and Clinical Molecular Biology (SIBioC) for reaching tentative consensus about the most informative diagnostic tests in emergency settings.
\end{abstract}

Methods: A survey, containing the most commonly ordered urgent laboratory tests and the relative clinical

\footnotetext{
altalian Society of Clinical Biochemistry and Laboratory Medicine (SIBioC-Laboratory Medicine)

${ }^{b}$ Academy of Emergency Medicine and Care (AcEMC)

*Corresponding author: Prof. Giuseppe Lippi, Section of Clinical Biochemistry, University Hospital of Verona, P.le LA Scuro 10, 37134 Verona, Italy, E-mail: giuseppe.lippi@univr.it; ulippi@tin.it. http://orcid.org/0000-0001-9523-9054

Mauro Panteghini: Department of Biomedical and Clinical Sciences “Luigi Sacco", University of Milan Medical School, Milan, Italy

Sergio Bernardini: Department of Experimental Medicine and Surgery, Division of Clinical Biochemistry, University of Rome Tor Vergata, Rome, Italy Laura Bonfanti and Gianfranco Cervellin: Emergency Department, Academic Hospital of Parma, Parma, Italy

Paolo Carraro: Department of Laboratory Medicine, ULSS3

Serenissima, Mestre-Venezia, Italy

Ivo Casagranda: Emergency Department, "Santi Antonio e Biagio e Cesare Arrigo" General Hospital, Alessandria, Italy
}

indications, was disseminated to eight relevant members of AcEMC and eight relevant members of SIBioC. All contributors were asked to provide numerical scores for the different laboratory parameters, where 1 indicated "strongly recommended", 2 "recommended in specific circumstances", and 3 "strongly discouraged". The mean results of the survey were presented as the mean of responders' values, and the parameters were finally classified as "strongly recommended" (mean value, 1.001.49), "weakly recommended" (mean value, 1.50-1.99), "discouraged" (mean value, 2.00-2.49) and "strongly discouraged" (mean value, 2.50-3.00).

Results: The results of the survey allowed defining a hierarchy of priority, wherein 24 tests were "strongly recommended". The use of five common tests was instead "strongly discouraged". For 16 additional parameters in

Mario Cavazza: Emergency Department, University Hospital “S. Orsola-Malpighi”, Bologna, Italy

Ferruccio Ceriotti: Ceriotti Ferruccio - Servizio di Medicina di Laboratorio, Ospedale San Raffaele, Milan, Italy

Marcello Ciaccio: Section of Clinical Biochemistry and Clinical Molecular Medicine, Department of Biopathology and Medical Biotechnology, University of Palermo - U.O.C. Laboratory Medicine, Policlinico University Hospital, Palermo, Italy

Daniele Coen: Emergency Departement, Niguarda Metropolitan Hospital, Milan, Italy

Davide Giavarina: Clinical Laboratory, St. Bortolo Hospital, Vicenza, Italy Fabrizio Giostra: Emergency Department, ASUR Marche Area Vasta 4 Fermo, Italy

Ciro Paolillo: Emergency Department, Academic Hospital of Udine, Udine, Italy

Mario Plebani: Department of Laboratory Medicine, University of Padova, Padova, Italy. http://orcid.org/0000-0002-0270-1711 Giorgio Ricci: Emergency Department, University Hospital of Verona, Verona, Italy 
the list, the consensus ranged between "weakly recommended" and "discouraged".

Conclusion: We hope that results presented in this joint AcEMC-SIBioC consensus document may help harmonizing panel of tests and requesting patterns in emergency setting, at least at a national level.

Keywords: consensus document; emergency medicine; laboratory testing; urgent testing.

\section{Introduction}

Despite it is now undeniable that laboratory tests are central to clinical decision making in patients with both acute and chronic conditions, the appropriateness of test ordering remains crucial. The large number of tests that become available in modern clinical laboratories thanks to automation may erode human and economic resources, especially when their prescription does not fulfill evidence-based criteria [1] or else inappropriate analyses may trigger additional, unjustified, and even invasive investigations when eventually generating the so-called laboratory incidentalomas (i.e. abnormalities detected by coincidence, without clinical symptoms or suspicion) [2]. Notably, it was also shown that the median length of stay (LOS) in the emergency department (ED), even in a very developed setting, may increase by up to $10 \mathrm{~min}$ for every five additional tests ordered, and by up to $17 \mathrm{~min}$ for each 30-min increase in turnaround time [3].

Reliable evidence attests that urgent testing represents a large part of laboratory activity, with median percentage of stat analyses approximating 33\% of all tests performed in clinical laboratories [4]. Additional evidence suggests that the panels of tests made available to the emergency physicians are extremely heterogeneous and varied worldwide, so highlighting a rather poorly standardized scenario [5, 6]. A multicenter Spanish study involving 36 hospitals showed that the rate of request of stat tests ranged from 44 to 412 per 1000 ED patient admissions [7]. The same study showed that the Spanish hospitals would have saved up to 1,000,000 unnecessary tests if every single laboratory would have achieved the appropriate indicator [7].

The mainstay of patient-oriented laboratory testing in the ED encompasses the selection of number and type of tests according to solid criteria of appropriateness. Despite some reliable guidance about appropriate laboratory testing in the ED has been provided by the Italian Society of Clinical Biochemistry and Clinical Molecular Biology (SIBioC-Laboratory Medicine; SIBiOC) [8], both
SIBioC and the Academy of Emergency Medicine and Care (AcEMC) recognized the need to reach a tentative consensus about the panel of tests that could be considered more informative, and hence appropriate, in specific emergency conditions in both children and adult populations.

\section{Materials and methods}

The board of the two societies identified eight members each, to whom a questionnaire containing a comprehensive list of the most commonly performed urgent laboratory tests and the relative clinical indications was administered. Briefly, the survey was disseminated by sending personal emails to eight relevant members of AcEMC and eight relevant members of SIBioC, providing a short deadline for collecting responses. All contributors were asked to indicate a numerical value for each of the different laboratory parameters included in the questionnaire, in which 1 indicated "strongly recommended", 2 indicated "recommended in exceptional circumstances" and 3 indicated "strongly discouraged". The results of the survey were then pooled and analyzed by calculation of the mean and standard deviation (SD) of all replies. Differences between AcEMC and SIBiOC replies were analyzed with Student's t test (Analyse-it, Analyse-it Software Ltd,). The scored parameters were finally classified as follows: mean value between 1.00 and 1.49 , strongly recommended; mean value between 1.50 and 1.99, weakly recommended; mean value between 2.00 and 2.49, discouraged; mean value between 2.50 and 3.00, strongly discouraged.

\section{Results}

The results of the survey are shown in Table 1, including the mean values $( \pm \mathrm{SD})$ and the statistical significance between SIBioC and AcEMC responders. Twenty-four parameters received such a high priority score, so that they could be defined as "strongly recommended". For two of these (i.e. total creatine kinase [CK] in trauma patients and D-dimer for diagnosing venous thromboembolism), the difference of values assigned by the members of the two societies reached statistical significance, but only for total CK the priority was finally different between SIBioC and AcEMC (strongly recommended for SIBioC and recommended for AcEMC, respectively). The consensus could be listed as satisfactory (i.e. p-value comprised between 0.05 and 0.50 ) or optimal (i.e. p-value $>0.50$ ) for 
Table 1: Summary of scores for laboratory testing proposed for use in the emergency department. Overall, Italian Society of Clinical Biochemistry and Laboratory Medicine (SIBioC) and Academy of Emergency Medicine and Care (AcEMC) mean values (standard deviation) are reported.

\begin{tabular}{|c|c|c|c|c|c|}
\hline Parameters & Overall & SIBioC & ACEMC & p-Value ${ }^{a}$ & Recommendation \\
\hline \multicolumn{6}{|l|}{ Anemia } \\
\hline Hemoglobin & $1.00(0.00)$ & $1.00(0.00)$ & $1.00(0.00)$ & 1.00 & Strongly recommended \\
\hline Hematocrit & $1.19(0.53)$ & $1.00(0.00)$ & $1.38(0.70)$ & 0.09 & Strongly recommended \\
\hline Red blood cell (RBC) count & $1.06(0.24)$ & $1.13(0.33)$ & $1.00(0.00)$ & 0.17 & Strongly recommended \\
\hline Mean corpuscular volume (MCV) & $1.13(0.33)$ & $1.25(0.43)$ & $1.00(0.00)$ & 0.07 & Strongly recommended \\
\hline RBC distribution width (RDW) & $2.00(0.87)$ & $2.25(0.83)$ & $1.75(0.83)$ & 0.14 & Discouraged \\
\hline \multicolumn{6}{|l|}{ Bleeding } \\
\hline Prothrombin time (PT) & $1.06(0.24)$ & $1.00(0.00)$ & $1.13(0.33)$ & 0.17 & Strongly recommended \\
\hline Activated partial thromboplastin time (APTT) & $1.06(0.24)$ & $1.00(0.00)$ & $1.13(0.33)$ & 0.17 & Strongly recommended \\
\hline Fibrinogen & $1.56(0.50)$ & $1.13(0.33)$ & $2.00(0.00)$ & $<0.001$ & Weakly recommended \\
\hline Platelet count & $1.00(0.00)$ & $1.00(0.00)$ & $1.00(0.00)$ & 1.00 & Strongly recommended \\
\hline \multicolumn{6}{|l|}{ Venous thromboembolism } \\
\hline D-dimer & $1.25(0.01)$ & $1.00(0.00)$ & $1.50(0.50)$ & 0.01 & Strongly recommended \\
\hline \multicolumn{6}{|l|}{ Acute pancreatitis } \\
\hline Amylase (total) & $2.50(0.87)$ & $2.75(0.66)$ & $2.25(0.97)$ & 0.14 & Discouraged \\
\hline Amylase (pancreatic) & $2.00(0.71)$ & $1.88(0.78)$ & $2.13(0.60)$ & 0.26 & Discouraged \\
\hline Lipase & $1.25(0.43)$ & $1.25(0.43)$ & $1.25(0.43)$ & 0.50 & Strongly recommended \\
\hline \multicolumn{6}{|l|}{ Liver disease } \\
\hline Alanine aminotransferase (ALT) & $1.00(0.00)$ & $1.00(0.00)$ & $1.00(0.00)$ & 1.00 & Strongly recommended \\
\hline Aspartate aminotransferase (AST) & $1.81(0.81)$ & $2.13(0.78)$ & $1.50(0.71)$ & 0.07 & Weakly recommended \\
\hline$\gamma$-Glutamyl transferase (GGT) & $2.06(0.56)$ & $2.25(0.66)$ & $1.88(0.33)$ & 0.10 & Discouraged \\
\hline Bilirubin (total) & $1.25(0.56)$ & $1.13(0.33)$ & $1.38(0.70)$ & 0.20 & Strongly recommended \\
\hline Bilirubin (conjugated) & $1.94(0.56)$ & $1.88(0.33)$ & $2.00(0.71)$ & 0.34 & Weakly recommended \\
\hline \multicolumn{6}{|l|}{ Acute myocardial infarction } \\
\hline Cardiac troponin I or T (cTnI or cTnT) & $1.00(0.00)$ & $1.00(0.00)$ & $1.00(0.00)$ & 1.00 & Strongly recommended \\
\hline Creatine kinase MB (CK-MB) & $2.69(0.58)$ & $3.00(0.00)$ & $2.38(0.70)$ & 0.02 & Strongly discouraged \\
\hline Myoglobin & $2.94(0.24)$ & $2.88(0.33)$ & $3.00(0.00)$ & 0.17 & Strongly discouraged \\
\hline Natriuretic peptides & $2.25(0.56)$ & $1.88(0.33)$ & $2.63(0.48)$ & $<0.01$ & Discouraged \\
\hline \multicolumn{6}{|l|}{ Renal disease } \\
\hline Creatinine & $1.00(0.00)$ & $1.00(0.00)$ & $1.00(0.00)$ & 1.00 & Strongly recommended \\
\hline Estimated glomerular filtration rate (eGFR) & $1.63(0.78)$ & $1.50(0.71)$ & $1.75(0.33)$ & 0.28 & Weakly recommended \\
\hline Urea & $1.50(0.50)$ & $1.75(0.43)$ & $1.25(0.43)$ & 0.02 & Weakly recommended \\
\hline Cystatin C & $2.69(0.46)$ & $2.63(0.48)$ & $2.75(0.43)$ & 0.31 & Strongly discouraged \\
\hline Neutrophil gelatinase-associated lipocalin & $2.25(0.66)$ & $2.13(0.78)$ & $2.38(0.48)$ & 0.24 & Discouraged \\
\hline \multicolumn{6}{|l|}{ Muscle trauma } \\
\hline Creatine kinase (CK), total & $1.38(0.60)$ & $1.00(0.00)$ & $1.75(0.66)$ & $<0.01$ & Strongly recommended \\
\hline Myoglobin & $1.81(0.81)$ & $1.38(0.70)$ & $2.25(0.66)$ & 0.02 & Weakly recommended \\
\hline Creatinine & $1.00(0.00)$ & $1.00(0.00)$ & $1.00(0.00)$ & 1.00 & Strongly recommended \\
\hline Lactate dehydrogenase (LDH) & $2.50(0.61)$ & $2.38(0.70)$ & $2.63(0.48)$ & 0.22 & Strongly discouraged \\
\hline \multicolumn{6}{|l|}{ Infection } \\
\hline White blood cell count (WBC) & $1.00(0.00)$ & $1.00(0.00)$ & $1.00(0.00)$ & 1.00 & Strongly recommended \\
\hline C-reactive protein & $1.13(0.33)$ & $1.00(0.00)$ & $1.25(0.43)$ & 0.07 & Strongly recommended \\
\hline Procalcitonin & $1.81(0.53)$ & $1.63(0.70)$ & $2.00(0.00)$ & 0.09 & Weakly recommended \\
\hline Presepsin & $2.88(0.33)$ & $2.88(0.33)$ & $2.88(0.33)$ & 0.50 & Strongly discouraged \\
\hline Lactate & $1.19(0.39)$ & $1.13(0.33)$ & $1.25(0.43)$ & 0.28 & Strongly recommended \\
\hline \multicolumn{6}{|l|}{ Ion imbalance } \\
\hline Calcium (total) & $1.50(0.61)$ & $1.13(0.33)$ & $1.88(0.60)$ & 0.01 & Weakly recommended \\
\hline Calcium (ionized) & $1.25(0.43)$ & $1.13(0.33)$ & $1.38(0.48)$ & 0.14 & Strongly recommended \\
\hline Magnesium & $1.94(0.66)$ & $2.13(0.78)$ & $1.75(0.43)$ & 0.14 & Weakly recommended \\
\hline Sodium & $1.00(0.00)$ & $1.00(0.00)$ & $1.00(0.00)$ & 1.00 & Strongly recommended \\
\hline Chloride & $1.06(0.24)$ & $1.00(0.00)$ & $1.13(0.33)$ & 0.17 & Strongly recommended \\
\hline Potassium & $1.00(0.00)$ & $1.00(0.00)$ & $1.00(0.00)$ & 1.00 & Strongly recommended \\
\hline Blood gas analysis & $1.00(0.00)$ & $1.00(0.00)$ & $1.00(0.00)$ & 1.00 & Strongly recommended \\
\hline \multicolumn{6}{|l|}{ Diabetes } \\
\hline Glucose & $1.00(0.00)$ & $1.00(0.00)$ & $1.00(0.00)$ & 1.00 & Strongly recommended \\
\hline Hemoglobin $A_{1 c}$ & $2.69(0.46)$ & $2.88(0.33)$ & $2.50(0.50)$ & 0.06 & Strongly discouraged \\
\hline Osmolality & $1.50(0.61)$ & $1.50(0.71)$ & $1.50(0.50)$ & 0.50 & Weakly recommended \\
\hline
\end{tabular}

aDifference between SIBioC and AcEMC. Bold values identify tests "Strongly recommended". 
the remaining 22 "strongly recommended" tests (Table 1). For the other parameters mentioned in the survey, the final consensus ranged between "weakly recommended" and "discouraged", whereas high consensus was found for "strongly discouraging" the use of $\mathrm{CK}$ isoenzyme $\mathrm{MB}$ and myoglobin for diagnosing acute myocardial infarction, cystatin $\mathrm{C}$ for diagnosing renal disease, presepsin in patients with suspected infections and hemoglobin $A_{1 c}$ for evaluating glycemic status. The range of recommendations for the other parameters was comprised between "weakly recommended" (10 tests) and "discouraged" (six tests) (Table 1). Among those, a disagreement between AcEMC and SIBioC was found for fibrinogen ( $p<0.001$; strongly recommended for SIBioC and discouraged for AcEMC), CK-MB ( $p=0.02$; strongly discouraged for SIBioC and discouraged for AcEMC), natriuretic peptides $(p<0.01$; weakly recommended for SIBioC and discouraged for AcEMC), urea ( $p=0.02$; recommended for SIBioC and strongly recommended for AcEMC), myoglobin in trauma ( $p=0.02$; strongly recommended for SIBioC and discouraged for AcEMC) and total calcium ( $p=0.01$; strongly recommended for SIBioC and weakly recommended for AcEMC).

\section{Discussion}

Previous evidence emphasized that the pattern of urgent test requesting in emergency settings is highly variable [7], thus underpinning the need to reach a more widespread consensus for harmonizing requesting attitudes. The main drivers for prescribing urgent laboratory tests in emergency settings entails obtaining data that may actually imply a substantial change in the diagnostic reasoning and/or managed care, combined with a low turnaround time (TAT), so that a timely intervention can be effectively established [9]. Rather understandably, inappropriateness of test ordering may have a dramatic impact on both laboratory organization and patients care. In the former case, excessive diagnostic testing may produce a negative impact on laboratory efficiency, by increasing the TAT, but also unnecessarily enhancing laboratory expenditures. More importantly, patient outcome may also be affected, since performing a large volume of inappropriate tests may either impact turnaround time for patients needing urgent results or potentially produce some laboratory "incidentalomas", which may then trigger unjustified, expensive and even invasive investigations [10].

The term appropriateness in laboratory medicine conventionally implies the optimization of human and economic resources, contextually offering the most useful data for improving outcomes and maintaining a high level of safety [11]. Implicit within this concept is that "inappropriateness" may comprise the underutilization of diagnostic tests, which may potentially lead to underdiagnosis or delayed diagnosis when essential laboratory analyses are overlooked [12].

A promising approach for enhancing appropriateness of urgent testing encompasses obtaining widespread consensus between emergency physicians and laboratory professionals about a list of informative tests according to specific emergency conditions. This consideration has prompted the AcEMC and SIBioC to merge their efforts and try identifying a potential list of urgent tests that can be recommended in ED patients with the most frequently diagnosed pathologies. Our survey, disseminated to eight relevant members of either society have allowed to identify of a group of 24 high priority tests, which were defined as "strongly recommended", as well as assigning low priority to five other common tests whose requesting should be defined as "strongly discouraged" (Table 1). Sixteen additional tests were either ranked as "weakly recommended" or "discouraged", so leaving room for further discussion about their implementation. This particularly refers to the 10 parameters scored as "weakly recommended" by the surveyed AcEMC-SIBioC representatives.

Along with the tests for which a general consensus was reached by the responders of the survey, other tests have necessarily to be added to the offered panel, since they allow the diagnosis and/or monitoring of certain emergency conditions. This refers, for instance, to cerebrospinal fluid (CSF) analysis for diagnosing infections and other acute disorders of the central nervous system (i.e. subarachnoid haemorrhage, acute neuroimmunological disorders, intracranial hypertension), serum $\beta$-HCG in the case of suspected pregnancy, specific drug testing (e.g. anticonvulsants, direct oral coagulation inhibitors), drug of abuse screening (including ethanol) and carboxyhemoglobin measurement for suspect poisoning or intoxication. Supplementary tests to the basic list that were indicated by one or more responders included ammonia, coagulation protein $\mathrm{C}$, antithrombin, thyroid-stimulating hormone (TSH), surface antigen of the hepatitis B virus (HBsAg), hepatitis B core antibody (HBcAb), malaria testing, reticulocyte count, blood culture, indirect Coombs test, estimate of cardiac troponin slope, serum albumin and white blood cell (WBC) differential.

Although routine emergency medicine test requesting does not affect efficiency of laboratory services, coincident multiple emergencies may disrupt the ability to timely provide test results, so influencing patient management and extending the LOS in short stay units. We hope 
that this joint AcEMC-SIBioC consensus report will help harmonizing the panel of tests and the requesting patterns in emergency settings, at least at the national level. Author contributions: All the authors have accepted responsibility for the entire content of this submitted manuscript and approved submission. As a result of an agreement among the editors, this article will be contextually submitted for publication in Clinical Chemistry and Laboratory Medicine, in Biochimica Clinica, the official journal of the Italian Society of Clinical Biochemistry and Laboratory Medicine (SIBioC-Medicina di Laboratorio), and in Emergency Care Journal, the official journal of the Academy of Emergency Medicine and Care (AcEMC).

Research funding: None.

Employment or leadership: None declared.

Honorarium: None declared.

Competing interests: The funding organization(s) played no role in the study design; in the collection, analysis, and interpretation of data; in the writing of the report; or in the decision to submit the report for publication.

\section{References}

1. Lippi G, Plebani M. Laboratory economics. Risk or opportunity? Clin Chem Lab Med 2016;54:1701-3.
2. Lippi G, Plebani M. Laboratory “incidentalomas”: facts or fiction? Eur J Intern Med 2010;21:572.

3. Li L, Georgiou A, Vecellio E, Eigenstetter A, Toouli G, Wilson R, et al. The effect of laboratory testing on emergency department length of stay: a multihospital longitudinal study applying a cross-classified random-effect modeling approach. Acad Emerg Med 2015;22:38-46.

4. Lippi G, Mattiuzzi C, Plebani M. Stat testing utilization in clinical laboratories. National survey of Italian Society of Clinical Biochemistry and Molecular Biology (SIBioC). Clin Chem Lab Med 2014;52:e79-84.

5. Hardin E. Emergency medicine and the laboratory. J Natl Med Assoc 1996;88:279-82.

6. Lippi G, Cervellin G, Plebani M. The ten commandments of laboratory testing for emergency physicians. Clin Chem Lab Med 2014;52:183-7.

7. Salinas M, Lòpez-Garrigòs M, Uris J. Differences in laboratory requesting patterns in emergency departments in . Ann Clin Biochem 2013;50:353-9.

8. Carraro P. Recommendations for appropriate laboratory tests in emergency department typical conditions. Biochim Clin 2011;35:207-28.

9. Smellie WS. Appropriateness of test use in pathology: a new era or reinventing the wheel? Ann Clin Biochem 2003;40:585-92.

10. Plebani M, Lippi G. Closing the brain-to-brain loop in laboratory testing. Clin Chem Lab Med 2011;49:1131-3.

11. Lippi F, Favaloro EJ. Exploring the iceberg of inappropriateness in hemostasis testing [editorial]. Diagnosis 2017;4:1-2.

12. Ferraro S, Panteghini M. The role of laboratory in ensuring appropriate test requests. Clin Biochem. 2017. doi: 10.1016/j. clinbiochem.2017.03.002. [Epub ahead of print]. 